\title{
The Quantum Handshake: An Electric Charge/Transactional Interpretation of the Single Electron Double-Slit Experiment
}

\author{
Eric Bond \\ Whitman College, Walla Walla, WA, USA \\ Email: enrique5043@outlook.com
}

How to cite this paper: Bond, E. (2021) The Quantum Handshake: An Electric Charge/Transactional Interpretation of the Single Electron Double-Slit Experiment. Journal of Quantum Information Science, 11, 71-83.

https://doi.org/10.4236/jqis.2021.112006

Received: April 6, 2021

Accepted: June 15, 2021

Published: June 18, 2021

Copyright $\odot 2021$ by author(s) and Scientific Research Publishing Inc. This work is licensed under the Creative Commons Attribution International License (CC BY 4.0).

http://creativecommons.org/licenses/by/4.0/

\begin{abstract}
A new and falsifiable realist interpretation of quantum mechanics is examined in relation to the sum over histories concept, pilot wave theory and the many-worlds interpretation. This electric charge/transactional model explains how the single electron double-slit experiment produces extremely localized endpoints from diffracted wavicles, why these endpoints are scattered around the entire surface of the absorber screen, and why these points of contact result in the characteristic fringe pattern as they accumulate. Advanced waves and substantive electric charge effects in the double-slit experiment are postulated, then this hypothesis is supported by a quantitative analysis of electron emission in comparison to lightning. The wider implications if advanced waves and electric charge distribution prove to be significant factors in the double-slit experiment are discussed, including possible parallels with meteorological and neurological phenomena.
\end{abstract}

\section{Keywords}

Double-Slit Experiment, Wave/Particle Duality, Interpretations of Quantum Mechanics, Wheeler-Feynman Absorber Theory, Advanced Wave, Retarded Wave, Lightning Bolt Mechanism,

Electric Charge, Neuroscience

\section{Basics of the Double-Slit Experiment}

The double-slit experiment ejects nanoscale particles from an emission device and towards a screen that records their final position as a florescent spot while it absorbs them. On the way, the particles pass through two slits. If the absorber screen is placed far enough behind the double slits, emission generates what 
seems to be an interference pattern, as if the particles are waves, but this effect only emerges from hundreds of particles, for each one contacts the screen at a specific point, as if not a wave. An interference pattern has shown up with photons, electrons, even molecules of as many as two thousand atoms. It works when streams of particles are emitted, and at least in the case of photons and electrons, when one particle is emitted at a time, so physicists have postulated that matter involves an intrinsic wave/particle duality [1]. All sorts of subatomic particles, atoms and molecules seem in actuality to be wave packets or "wavicles".

It is easy to imagine a stream of wavicles interfering as they diffract through the slits to yield an array of light and dark bands on the florescent screen corresponding to in phase and out of phase waves. This would resemble the classic experiment performed in the 19th century, where a beam of light was diffracted by a single aperture to then pass through double slits as a spreading field which apparently interfered with itself to similar result [1].

Interference patterns from one at a time particle emission are a more difficult outcome to account for. A typical explanation is that the wavicle passes through both slits to interfere with itself, spreading in the double-slit chamber and then spontaneously collapsing in some way upon contact with the absorber surface to give a particulate signature [1]. This wave function collapse mechanism is quite the brain teaser: does the wavicle fan out invisibly after it diffracts and then somewhat mystically end up at a very localized endpoint? Why would many localized endpoints with no likeness to waves at all look like in phase and out of phase waves as they accumulate on the absorber screen? What exactly is going on?

This gets even trickier when a sensor is placed at one of the slits during the single wavicle, two slit experiment in order to detect transmission. The wavicle is detected $50 \%$ of the time, so there is an equal (and unpredictable) chance of it passing through either slit in any single trial. If a sensor is present, the interference pattern dissolves and wavicles engender two narrow bands on the florescent screen, as if they were never a wave [1]. This led researchers to propose a decoherence mechanism: diffuseness of the wavicle, according to this account, is disrupted by interaction with large collections of tightly knit atoms such as exist within the sensor or absorber screen. Wavicles supposedly assume a more localized form that obeys principles of Newtonian mechanics as they jostle amongst themselves in vast quantities [2].

The state of an individual wavicle combines position with momentum, and the exact values for these two properties cannot be known simultaneously, at least in the vast majority of extant cases, because of fundamental indeterminacy intrinsic to associated math, the Heisenberg uncertainty principle. The more precisely one knows the position, the less precisely one knows the momentum, and vice versa [3]. Yet we have techniques that can definitively project the behavior of wavicles in bulk. Heisenberg et al's matrix mechanics is a purely algebraic method that outputs an accurate range of probabilities based on initial conditions of a wavicle [4]. Schrodinger's wave function also attains correct re- 
sults by representing emission as evolving through space in a continuous manner to determine a quantum system's statistical parameters [5].

Some physicists have attempted to dig deeper and explain the enigma of an individual wavicle's behavior in the double-slit experiment. Unfolding of the emitted wavicle's course has been theorized as a quantitative combo of most and least probable processions preceding contact with the absorber, a path integral formulation which models all possible trajectories as jointly influencing the endpoint [6]. This concept of many trajectories is reified in Bohmian mechanics, which regards a nanoscale wavicle as more particulate than wavelike, riding the flow of invisible pilot waves hypothesized to span the whole double-slit chamber and set the distribution of endpoints via constructive and destructive interferences [7]. It has even been proposed that all possible trajectories are actually occupied, with the wavicle being in a spacetime-transcending superposition of perhaps infinite states unless measurement collapses the wave function, an event allegedly splitting reality into parallel universes where all possible outcomes are realized, the many-worlds interpretation [8].

Despite these realist adventures, Neil's Bohr's initial, antirealist Copenhagen interpretation of the 1920's persists as the most popular theory. It claims that statistics arising from large collections of wavicles are the limit of what can be predicted about a double-slit system, with probabilistic wave/particle duality indelibly stamped into the experimental context [9]. A definitive realist account of the double-slit experiment with a single wavicle continues to elude researchers.

The goal of this paper is not to robustly reconstitute underlying math nor present new experimental results, but rather propose a realist interpretation that seems to have thus far been neglected. Perhaps it is not sum over histories, pilot waves or parallel worlds that should be held responsible for the fringe pattern on absorber screens in the case of single electron emission. Instead, momentary electric charges along with advanced waves, both generated by the emission event, incidental to ejection of a wavicle, could permeate a space like the double-slit chamber and exert influence on nanoscale dynamics such as trajectory, at least in the case of negatively charged electrons and perhaps additional particles by some form of similarity. The following will describe what a realist mechanism of this kind might look like and outline ramifications if phenomena such as these prove efficacious in this context and then necessarily elsewhere.

\section{Flaws in Existing Models of the Double-Slit Experiment and a Possible Alternative}

Previous realist theories evince shortcomings. Sum over histories predicts the statistics of wavicle transmission through space in many situations, but is more a method of calculation than an observable phenomenon and takes no stance in relation to how closely its snapshots of wavicle trajectory conform to the state of matter itself.

Bohmian mechanics' image of guide waves that channel wavicles towards their destinations matches the math of quantum phenomena, including within 
its scope the apparent nonlocality of entanglement interpreted as driven by underlying wave fluctuations, but these waves have not been observed directly. Pilot waves fail to explain how an individual electron that clearly diffracts at the double-slits recombines to end up as an extremely localized point particle on the absorber screen. No known mechanism explains how a wavicle scattered at roughly six million meters per second culminates as a tiny dot, with each trial distributed randomly [10].

A many-worlds interpretation also fits the double-slit experiment's data, simply asserting that there is no collapse mechanism, but the parallel universes it expounds remain unobserved and it is not at all evident they even can be, so this theory has minimal prospect of modeling what happens to a solitary wavicle in our world. If distinct timelines verifiably influence or are influenced by ours, they will of necessity be part of our own sphere of causes and preclude the existence of many worlds, so this idea is not really setting forth anything falsifiable about existing quantum mechanics, rather claiming on the basis of a thought experiment that our framework may need to be revised in the future.

So for single wavicles passing through double slits, the realist currently possesses an agnostic model, a model based on unobserved and perhaps implausible principles, and a model which it is uncertain scientists will be able to prove true or false. The phenomena of pilot wave theory and the many-worlds interpretation have not been conclusively ruled out, but does an alternative exist?

Wheeler-Feynman absorber theory proposed that interactions between particles such as electrons occur both forwards and backwards in time. A model including time reversed signals between particles accounted for apparently instantaneous entanglement observed to take place at the quantum scale, a synchronization supposed emergent from the coupling of opposite motion through spacetime [6].

In 1986, this idea was adapted by John Cramer into a transactional interpretation of quantum mechanics, eventually applied to the double-slit experiment. In this schema, the forward in time emission event is conjoined to a backward in time signal from the absorber material, altogether generating interferences which variously adjust each particle's path such that a relatively broad pattern materializes on the florescent screen. He called the forward in time motion a retarded wave and the backward in time motion an advanced wave [1].

It has subsequently been demonstrated that even the most instantaneous processes are only near instantaneous, and minuscule delay in this case can be explained if the advanced wave is not absolutely time reversed in action at a distance, but instead rapidly ricochets around and through a space from high energy sources to instigate complex interference. In the double-slit experiment, these advanced waves as initiated by the energetic emission event might divert a wavicle along its path, steering it to a different location in each trial.

Then with all this agitation, why such symmetry in the absorber screen's interference pattern? Does the particle diffract through both slits, interfere with it- 
self, follow an indirect path under the influence of guide waves, then perhaps somehow collapse or otherwise induce a particlelike signature at a specific point when the energy of this process reaches an absorber? The traditional models are fraught with problems, for mechanisms which would be in effect have not been observed. But perhaps there is an alternative, at least in the electron experiment: these wavicles are negatively charged particles of very small mass $\left(9 \times 10^{-31} \mathrm{~kg}\right.$ [10]), and so though they are traveling at breakneck speed, it could prove possible for them to be impacted by electric charge. If charge distribution within the double-slit chamber affects electron trajectory, this might by some mechanism be responsible for the interference fringe on the absorber screen. So if we hypothesize that electric charge and advanced waves are substantive factors, how might this mechanism work?

\section{A Lightning Bolt Model of the Double-Slit Experiment}

The double-slit experiment with a single electron is performed using a cathode ray tube from which the wavicles are ejected [11]. This of course generates electric charge, the precise features of which seem not to have been thoroughly examined. The electron apparently diffracts through the slits like a wave and then resumes particlelike behavior downstream, converging on a seemingly random point as it contacts the absorber screen. Accumulation of hundreds of trials generates the fringe of alternating lighter and darker bands. The projectile electron's speed is huge, making it surprising that this diffracted wavicle maintains or regains structural integrity after collision with the slits, but it clearly does, so assuming the electron is not dividing into multiple universes, each with its own particle, what kind of force might be responsible for the phenomenon?

Whether electric charge distribution indeed influences electron behavior in the double-slit experiment is still very much in question, but nature at least gives us somewhere to begin. Cloud to ground lightning strikes seem to have similar properties: extremely fast rate of electron transmission as well as electric charge conditions which synchronize the behaviors of atoms in the cloud, ground, interposing space and lightning current itself. Could the single electron experiment resemble a lightning strike, with momentary electric charges induced by the emission event causing the wavicle to flow like a current between cathode ray gun and absorber? We can start to get a handle on this concept by analyzing the anatomy of lightning.

During a thunderstorm, numerous regions of like charge form in clouds and on the ground with an oppositely charged space between that is thousands of meters thick. In order for a lightning strike to happen, interposing opposite charge must reach tens of thousands of volts per inch. The lightning strike travels at 270,000 miles per hour, which comes to 120,700 meters per second or 0.1207 meters per microsecond. It averages 2.5 centimeters in width and 2.5 miles or 4023.36 meters in length ( 1 mile $=1609.344$ meters). The total time the lightning bolt requires to reach the ground is thus: 


$$
4023.36 \mathrm{~m} \times \frac{1 \mathrm{~s}}{120700 \mathrm{~m}}=0.0333 \mathrm{~s} .
$$

The lightning bolt initiates with a stepped leader, and each step is roughly 50 meters in length for a total of:

$$
\frac{4023.36 \mathrm{~m}}{50 \mathrm{~m}}=80 \text { steps . }
$$

The duration of each stepped leader: (extra digits beyond significant figures are sometimes retained for purposes of calculation or clarity, a caveat resolvable by precision measurement).

$$
50 \mathrm{~m} \times \frac{1 \text { micros }}{0.1207 \mathrm{~m}}=414.25 \text { micros . }
$$

Before the lightning strike terminates, a roughly 10 meter long return streamer snakes up from the ground and connects with the stepped leader. When the two meet, a surge of charge is transmitted. A dart leader then emerges from the cloud and smoothly follows the path blazed by the stepped leader at a faster rate [12] [13].

Why does a lighting bolt's first stroke of current proceed in $50 \mathrm{~m}$ steps? With inspiration from the transactional interpretation, we can hypothesize that these zigzagging changes in direction [14] occur due to a series of advanced waves generated by the lightning burst, invisibly ping ponging between a retarded wave, which includes the stepped leader, and the ground, diverting a lightning bolt's trajectory roughly 80 times. If we take a lightning strike as our standard, the rate at which an advanced wave travels is given by the following:

$$
\frac{2 \times 4023.36 \mathrm{~m}-50 \mathrm{~m}}{414.25 \text { micros }}=19.3 \mathrm{~m} / \text { micros . }
$$

Moving to the double-slit experiment, the space between cathode ray emitter and absorber screen in the single electron double-slit experiment is 2 meters, and as already mentioned, the electron travels at a more rapid rate than a lightning bolt, 6 million meters per second or 6 meters per microsecond. This means that the total time required for the electron to reach the absorber is:

$$
2 \mathrm{~m} \times \frac{1 \text { micros }}{6 \mathrm{~m}}=0.333 \text { micros . }
$$

In the transactional model, an electron's trajectory somehow depends on retarded/advanced wave interference. Then how would an advanced wave be affecting the double-slit experiment if we assume its rate of motion is the same as in the hypothesized case of a lightning bolt, $19.3 \mathrm{~m} / \mathrm{micros}$ ?

First, a summary of the data so far in addition to unknowns (Table 1):

We can calculate $x_{m}$ and $y_{\text {micros }}$ using three equations.

1) After initiation from the same emission source, the distance covered by a hypothetical advanced wave during the double-slit experiment divided by corresponding time elapsed until retarded/advanced wave interference, equaling the average rate of an advanced wave: 
Table 1. Known and unknown distances, times, rates, and quantity of steps for a lightning bolt and the double-slit experiment.

\begin{tabular}{ccc}
\hline & Lightning Bolt & Double-slit experiment \\
\hline Total distance & $4023.36 \mathrm{~m}$ & $d_{e}=2 \mathrm{~m}$ \\
Total time & $0.0333 \mathrm{~s}$ & $t_{e}=0.333$ micros \\
Rate of travel & $0.1207 \mathrm{~m} / \mathrm{micros}$ & $r_{e}=6 \mathrm{~m} / \mathrm{micros}$ \\
Distance per step & $50 \mathrm{~m}$ & $X_{m}$ \\
TIme per step & $400 \mathrm{micros}$ & $y_{\text {micros }}$ \\
Distance covered by first advanced wave & $8000 \mathrm{~m}$ & $2 \times 2 \mathrm{~m}-X_{m}$ \\
Average rate of advanced wave & $r_{a}=19.3 \mathrm{~m} / \mathrm{micros}$ & $r_{a}=19.3 \mathrm{~m} / \mathrm{micros}$ \\
Quantity of steps & 80 & $q_{s}$ \\
\hline
\end{tabular}

$$
\frac{2 d_{e}-x_{m}}{y_{\text {micros }}}=r_{a}
$$

2) The length of a step divided by duration of the step, which equals the rate of electron transmission:

$$
\frac{x_{m}}{y_{\text {micros }}}=r_{e}
$$

3) Solving Equation (2) for $y_{\text {micros }}$, then substituting into Equation (1) and solving for $x_{m}$ gives:

$$
x_{m}=0.47431 d_{e}
$$

For the initial retarded wave:

$$
x=0.47431 \times 2
$$

This equals $0.94862 \mathrm{~m}$, the distance traveled by the retarded wave before its first interference with the advanced wave, and solving Equation (2) for $y_{\text {micros }}$ using this value gives 0.15810 micros, the time elapsed. $d_{e}-x_{m}=2 \mathrm{~m}-0.94862 \mathrm{~m}$ $=1.05138 \mathrm{~m}$, the distance remaining between electron and absorber screen upon first interference. $t_{e}-y_{\text {micros }}=0.333$ micros -0.15810 micros $=0.17490$ micros, the time remaining before the electron reaches the absorber.

The initial $d_{e}-x_{m}$ value becomes $d_{e}, 1.05138 \mathrm{~m}$, and is entered into Equation (3) to determine the next $x_{m}$ value, $0.49868 \mathrm{~m}$. Substitution of this new $x_{m}$ into Equation (2) and solving for $y_{\text {micros }}$ gives $8.3113 \times 10^{-2}$ micros. Subtracting the new $x_{m}$ from $d_{e}$ of the first interference yields $d_{e}$ for the the second interference, $0.55270 \mathrm{~m}$, and $y_{\text {micros }}$ subtracted from $t_{e}$ of the first interference yields $t_{e}$ for the second interference, $9.1787 \times 10^{-2}$ micros. This procedure is repeated to calculate values for all further interferences, which are summarized in Table 2.

At this point, approximately less than $6.9256 \times 10^{-4}$ microseconds until transmission is complete, the retarded wave might meet a return streamer from the absorber screen, which if proportional to a lightning bolt's is $4.97 \mathrm{~mm}$ long, and the quantum handshake surge occurs, with the electron's current channeled to a specific location, making a florescent spot. It is preliminarily hypothesized that this current consists in around $q_{s}=9$ steps or angled deviations along its course, diverting it to a varied range of locations on the absorber. 
Table 2. $x_{m}, d_{e}-x_{m}, y_{\text {micros }}$, and $t_{e}-y_{\text {micros }}$ values for successive interferences.

\begin{tabular}{ccccc}
\hline Interferences & $x_{m}$ & $d_{e}-x_{m}$ & $y_{\text {micros }}$ & $t_{e}-y_{\text {micros }}$ \\
\hline 1st & 0.94862 & 1.05138 & 0.15810 & 0.17490 \\
2nd & 0.49868 & 0.55270 & $8.3113 \times 10^{-2}$ & $9.1787 \times 10^{-2}$ \\
3rd & 0.26215 & 0.29055 & $4.3692 \times 10^{-2}$ & $4.8095 \times 10^{-2}$ \\
4th & 0.13781 & 0.15274 & $2.2968 \times 10^{-2}$ & $2.5127 \times 10^{-2}$ \\
5th & $7.2446 \times 10^{-2}$ & $8.0294 \times 10^{-2}$ & $1.2074 \times 10^{-2}$ & $1.3053 \times 10^{-2}$ \\
6th & $3.8084 \times 10^{-2}$ & $4.2210 \times 10^{-2}$ & $6.3474 \times 10^{-3}$ & $6.7056 \times 10^{-3}$ \\
7 th & $2.0021 \times 10^{-2}$ & $2.2189 \times 10^{-2}$ & $3.3368 \times 10^{-3}$ & $3.3688 \times 10^{-3}$ \\
8th & $1.0524 \times 10^{-2}$ & $1.1665 \times 10^{-2}$ & $1.7541 \times 10^{-3}$ & $1.6147 \times 10^{-3}$ \\
9th & $5.5328 \times 10^{-3}$ & $6.1322 \times 10^{-3}$ & $9.2214 \times 10^{-4}$ & $6.9256 \times 10^{-4}$ \\
\hline
\end{tabular}

Despite a wide diversity of endpoints, with each individual instance seeming effectively random at this stage of knowledge, the symmetry of lighter and darker bands can be explained as a product of symmetrical charge. During a storm, charge positions in clouds and on patches of ground are in chaotic flux, so that lighting strikes do not display an easily discernible pattern, even as an average of hundreds. But in a double-slit experiment, the emitter and slits are arranged so that the apparatus has bilateral symmetry, and this makes the emission charge, patches of charge along the absorber surface, and charges in interposing space likewise symmetrical, distributed in a mathematically recursive way between strong and weak. High velocity of the electron combined with diffraction and advanced wave collision would tend to knock the wavicle away from evenly spaced regions of most concentrated charge on the absorber, but given enough time the electron's destination is slightly more likely to end up near stronger charges. Possibly for this reason, hundreds of trials result in an on average closer proximity to greater electric charge and an orderly pattern alternating between high and low density of florescent spots.

So the overall causality of this lightning bolt model can be outlined as follows:

Some moments after the first retarded wave/current is formed, involving something comparable to the stepped leader produced by a thunder cloud, an advanced wave originating from the same emission arrives, which was mostly reflected off the absorber. Retarded and advanced wave interfere to deviate the trajectory of the electron and current along their forward path. This first advanced/retarded wave interference partially dissipates in the emitter's direction to negligible effect, but a proportion continues on towards the absorber due to constructive interference, as a retarded wave pacing the electron and an advanced wave outpacing it. The electron and its current rapidly close their distance to the absorber in a stairstepping sequence of zigzagging deviations induced by successive interferences. At some point a prong of current emerges from the absorber, like a return streamer, and its effect is ultimately to draw the retarded wave/current, still somewhat diffuse, into a particular spot on the ab- 
sorber surface. Leader/streamer contact is made and the quantum handshake occurs, a surge of charge briefly connecting the emitter and absorber directly, like a lightning bolt, in this case invisible. Each trial appears random, but symmetry of the experimental setup results in an organized pattern of alternation between high and low density of contact corresponding to strong and weak electric charge, a probability distribution which gives the appearance of an interference fringe.

Decoherence effects of the sensor are also explicable if the electron does not move in isolation, but gathers steam via interaction with the electric charges of interposing space such that it flows to the absorber within a retarded wave and current that are relatively large compared to the wavicle's mass and diameter. If a sensor is present, it would be dissolving the fringe pattern into two clustered bands directly behind each slit because it disrupts the electron entourage's horizontal diffraction as it spans the double slits. Vertical degrees of freedom, which likely correspond to relatively homogeneous verticality of the two nearest charge peaks on the absorber, do not seem to be inhibited. Visibility of the florescent dot appearing upon absorber contact suggests much more energy than that of merely an electron is transmitted. As for constitution of the current, a lightning bolt is roughly 2.5 centimeters thick, and scaled to dimensions of the double-slit experiment this amounts to a 12 micrometer thickness. If a flash of current approaching that width can be detected during electron transmission, this lends the model support.

Further corroboration would come from discovering electric charges in the double-slit chamber strong enough to interact with an electron or electron current. If something like a flicker, one or more strokes of current, or dart leader is observed, this also substantiates the model. Properties of a lightning bolt should be examined more closely to see if the steps of its step leader get smaller upon approaching the ground. This would intimate that advanced waves are causing interference.

It should be emphasized that most of the figures in these calculations are extremely approximate, but it at least seems reasonable that advanced waves and electrical energy may be exacting significant effects during the double-slit experiment. If charge distribution as well as size and shape of the retarded wave, advanced wave and electron current are proven to influence transmission, a systematic relationship between these and additional factors such as all of the experiment's constituent energies, masses, velocities and distances will no doubt be found, provisional of a formula integrating new and already established variables.

\section{Tentative Hypotheses about the Nature of Wave/Particle Interactions}

The retarded wave would include a flash of electrically charged current between emitter and absorber that morphs the wavicle's shape somehow via charge dynamics and within which the wavicle flows as a high energy cluster of matter, 
tightly knit enough to avoid being substantially absorbed by the environment as it travels to the florescent screen, and pliant enough to squeeze through the slit or slits. But what is an advanced wave? We can start by quickly outlining the nature of matter waves in general.

All matter so far seems capable of evincing quantum, wavelike properties, but in Earth's macroscopic objects that compose and discompose trillions upon trillions of thermally driven atomic bonds each instant, a process which is essentially an electromagnetic flux centered on the infrared portion of the spectrum, wavicles can for many purposes be regarded as more particlelike than quantumlike, for this is what classical particles as a certain kind of interaction between innumerable atoms are: emergent structures with properties substantially modelable in terms of "heat" or thermal energy.

If we were to launch a baseball at the screen, it would slow slightly due to macroatomic friction in the chamber and bounce off the slits rather than transmit through them because massive and dense size induces enough thermal equilibrium, a charge-canceling decoherence among its tangle of interfering atoms, that it cannot squeeze through nor respond to many effects pervading the subatomic scale, instead largely obeying laws which apply to relative atomic stability, those of classical physics.

Electromagnetic radiation $\left(3 \times 10^{8} \mathrm{~m} / \mathrm{s}\right)$ near the visible portion of the spectrum is a sweet spot in relationship to Earthlike molecules, constantly being emitted at relatively high intensities that saturate the environment. However, it is absorbed in very large amounts as well, which amongst the double-slit context and most Earthbound situations reduces average intensity over any macroatomic range of space such that negligible impact is had on outcomes. Some moderately sized radio waves may also be generated by high energy of the emission device, but exist at such low intensities within the double-slit context that they are readily absorbed by molecules and also have negligible effect.

At this point, two theories seem possible. Advanced waves $\left(2 \times 10^{7} \mathrm{~m} / \mathrm{s}\right)$ could be contiguous in some way with electromagnetism, perhaps arising at low intensities during an energetic emission event. Despite low intensity, they might be rapid and concentrated enough to subvert much inhibitance by molecules, reaching the absorber with their intensity almost undiminished. This might be enough to create waves that effectively ping pong between retarded waves and absorber, involved in steering the electron current's primarily linear motion. A rival hypothesis is more reminiscent of pilot wave theory, in which advanced waves are a distinct and separate form of matter underlying the type conventionally modeled by physics. These advanced waves would be participating in extremely fast perturbation while capable of interference with various forms of electromagnetic matter if the environmental trigger is suitably high enough in energy.

It is claimed that the double-slit experiment involves something like two energy peaks: an electron's retarded wave/current of lower speed, high but somewhat localized intensity, very low absorption, and the advanced wave's 
higher speed, low but concentrated intensity, similarly low absorption, separated by a sizable gap of relative speed, intensity and absorption that proves negligible. Earth's molecular structure cancels out the force of much motion in many situations, perhaps allowing some wavicles to interact with advanced waves, and the highly sensitive double-slit experiment with its minuscule lightning bolt of current containing a tiny, relatively isolated electron might be such a context.

\section{Wider Implications of the Electric Charge/Transactional Interpretation}

So sum over histories, pilot wave theory and the many-worlds interpretation are not the only options for explaining the double-slit experiment and quantum behavior in matter generally. It may be the case that an electric charge/transacttional interpretation can inform our understanding of quantum phenomena, modeling the synchronicity of electrical currents and charges alongside a complementary flow of advanced waves that interfere with themselves and with wavicles, electric currents and more to affect the motions of matter. This does not rule out previous interpretations, for pilot waves and the splitting of spacetime can still conceivably exist, but a verification of this new model via the double-slit experiment and in further domains could impart an additional layer of causality to our knowledge of the physical world.

Electric charge and current already play a role in elucidating many natural occurrences, notably the weather and the brain. It has been shown that there are strong parallels between the properties of lightning and neurons. The average energy density of action potentials which transmit current in the brain is similar to the global atmosphere as concentrated most heavily in thunderstorms: $10^{-7}$ watts per meter ${ }^{3}$. The current of a lightning stroke is comparable in cross-sectional area to an action potential. The average pulse rate of cloud to cloud and cloud to ground lightning combined is near an action potential's refractory period. The velocity of current in a lightning bolt relative to the mass of its constituent electrons is close to the velocity of an action potential relative to the mass of $\mathrm{Na}^{+}$ions which play the primary role in propagation along an axon's length. Rate of energy transfer within synaptic spaces, tied to the mass and motion of particles such as neurotransmitters, is equivalent to the rate of surge in electric current during contact between a stepped leader and return streamer. The effect of a single neuron's firing on the overall energy of brain waves is proportional to the effect of a lightning strike on energy of the global earth-ionospheric cavity within which electrical storms take place. And contours of variability in power density throughout the mass of a human brain as indicated by EEG are proximal to power density fluctuations as they take place in the earth-ionospheric cavity [15].

Copious evidence exists that electrical activity in the atmosphere matches much of what happens in the brain, and if lightning turns out to correlate with the double-slit experiment, then all of this-the weather, brain, and quintessen- 
tial demonstration of quantum mechanics by experiment-definitively shares new physical principles and already established physical principles in novel ways. It might be possible to correlate numerous variations on the double-slit experiment, in which electric charge properties, energies, masses, distances and velocities are modified, with neuroscience, meteorology and perhaps additional domains where dynamics of electrical potential and electric current are in effect. If advanced waves do in fact exist, their properties can be correlated across disciplines, and may be proven to play a part in brain function. That advanced waves, retarded waves and the electron travel at constant velocities is a big assumption, for these phenomena could be the product of a single wave complex perturbing at amorphously variable rate, but if the electric charge/transactional interpretation of quantum mechanics turns out justified as a subject of broader research, this will be testable in diverse settings. Multiple avenues for experimental investigation are available going forward.

\section{Conflicts of Interest}

The author declares no conflicts of interest regarding the publication of this paper.

\section{References}

[1] Gribbin, J. (2013) Erwin Schrodinger and the Quantum Revolution. John Wiley \& Sons, Inc., Hoboken.

[2] McFadden, J. (2014) Life on the Edge: The Coming of Age of Quantum Biology. Crown Publishers, New York.

[3] Britannica, Heisenberg Uncertainty Principle. https://www.britannica.com/science/quantum-mechanics-physics/Heisenberg-unce rtainty-principle

[4] Britannica, Matrix Mechanics. https://www.britannica.com/science/matrix-mechanics

[5] Britannica, Schrodinger's Wave Mechanics. https://www.britannica.com/science/quantum-mechanics-physics/Schrodingers-wa ve-mechanics

[6] Halpern, P. (2018) The Quantum Labyrinth: How Richard Feynman and John Wheeler Revolutionized Time and Reality. Hachette Book Group, Inc., New York.

[7] Falk, D. (2016) New Support for Alternative Quantum View. https://www.quantamagazine.org/pilot-wave-theory-gains-experimental-support-20 160516

[8] Stanford Encyclopedia of Philosophy (2014) Many-Worlds Interpretation of Quantum Mechanics. https://plato.stanford.edu/entries/qm-manyworlds

[9] Britannica, Copenhagen Interpretation. https://www.britannica.com/science/Copenhagen-interpretation

[10] Nuffield Foundation, The Speed of Electrons. https://spark.iop.org/speed-electrons

[11] Circuit Globe, Electron Gun. https://circuitglobe.com/electron-gun.html

[12] Zavisa, John, How Lightning Works. 
https://science.howstuffworks.com/nature/natural-disasters/lightning1.htm

[13] Britannica (2010) Pilot Streamer: Lightning Initiation. https://cdn.britannica.com/62/108862-004-90FE873D.jpg

[14] Patterson, R. (2010) Lightning in Super Slow Motion. https://www.youtube.com/watch?v=RLWIBrweSU8

[15] Persigner, M.A. (2012) Brain Electromagnetic Activity and Lightning: Potentially Congruent Scale-Invariant Quantitative Properties. Frontiers in Integrative Neuroscience, 6, 19. https://www.frontiersin.org/articles/10.3389/fnint.2012.00019/full https://doi.org/10.3389/fnint.2012.00019 